\title{
Gestión proactiva de crisis en el turismo: una experiencia de estudio en Galicia
}

\author{
Diego Rodríguez-Toubes Muñizi \\ José Antonio Fraiz Brea ${ }^{\text {ii }}$
}

Universidad de Vigo (España)

\begin{abstract}
Resumen: Los impactos potenciales de acontecimientos negativos que pueden afectar a destinos turísticos se han multiplicado en los últimos años. El turismo es un sector fragmentado y diverso por lo que es difícil establecer la forma de gestión de crisis que debería llevarse a cabo. Distintos modelos facilitan esta tarea centrándose especialmente en la preparación y planificación para conseguir una mejor coordinación entre los distintos agentes implicados en la resolución de la crisis. La planificación colaborativa es una forma de gestión de crisis poco utilizada y que genera una cultura comprometida con la resolución de problemas en tiempo de crisis. Una serie de entrevistas realizadas a responsables del turismo en Galicia parecen confirmar esta idea.
\end{abstract}

Palabras clave: Crisis; Gestión de desastres; Destino turístico; Planificación colaborativa; Gestión proactiva

Title: Proactive crisis management in tourism: a study experience in Galicia

Abstract: The potential impacts of negative events that may affect tourist destinations have multiplied in recent years. Tourism is a fragmented and diverse sector so is difficult to establish the form of crisis management should be carried out. Different models make this task easier with particular focus on the preparation and planning for better coordination between the various actors involved in resolution of the crisis. Collaborative planning is a form of crisis management rarely used and generates a culture committed to solving problems in times of crisis. A series of interviews with responsible tourism in Galicia seems to confirm this insight.

Keywords: Crisis; Disaster management; Tourist destination; Collaborative planning; Proactive management.

i Departamento de Organización de Empresas y Marketing. Facultad de Ciencias Empresariales y Turismo. Universidad de Vigo. Campus Ourense. E-mail: drtoubes@uvigo.es

ii Departamento de Organización de Empresas y Marketing. Facultad de Ciencias Empresariales y Turismo. Universidad de Vigo. Campus Ourense. E-mail: jafraiz@uvigo.es 


\section{Introducción}

El mundo actual es más propenso a sufrir crisis y desastres debido a la presencia de varios factores hasta ahora desconocidos o, al menos, no sufridos con tanta pertinacia: el crecimiento de la población, el desarrollo de las ciudades, la presión económica global, la degradación de la tierra y el deterioro medioambiental, la presencia de la guerra como amenaza global, y el impacto de la tecnología (Faulkner, 1999). Richardson (1994) en su trabajo sobre la gestión estratégica de la crisis y la necesidad de un aprendizaje sistemático en tiempos de crisis, parte de la base de que estamos en un mundo crisis-dependiente (crisis-ridden) o dicho de otra forma, propenso a la crisis. El entorno de los negocios, por su propia naturaleza, por la tecnología y la acción del hombre, se ha convertido en un entorno cada vez más complejo e inestable y que es necesario atender en tiempos de respuesta cada vez menores, realidades que Lerbinger (1997) considera como un indicio de que nos encontramos en una "era de crisis". McKercher y Young (1999) afirman que el turismo cada vez está más desarrollado tecnológicamente, debido a ello, los sistemas de comunicación, información y reserva son cada vez más sofisticados y a la vez más frágiles y vulnerables. En este sentido, los efectos de una crisis pueden ser más devastadores, y a su vez, la misma estructura del sistema es más propensa a la generación y propagación en cadena de las crisis.

El habitual emplazamiento de las actividades turísticas en localizaciones de más riesgo potencial se debe tener en cuenta para el establecimiento de las alertas de seguridad. Faulkner (2001) señala que el incremento del volumen mundial de la actividad turística ha ido acompañado con una mayor atracción por destinos exóticos con riesgo más alto para los turistas que los visitan. Con frecuencia los turistas son más vulnerables que los residentes a las situaciones de desastre porque son más dependientes, están menos familiarizados con los riesgos locales y los recursos que tienen a su disposición para evitarlos. Las crisis en los mercados de destino no se deben tratar como un problema confinado a unos específicos destinos en apariencia más conflictivos: es un hecho global (Beirman, 2003). WTO y WMO (1998: 1) lo expresan en los siguientes términos:

- El turismo es un fenómeno global que afecta a los movimientos de millones de personas en todo el mundo de todos los países

- El turismo se ha desarrollado con mucha frecuencia en zonas más expuestas a desastres naturales o, al menos, con más probabilidad de sufrirlos, como costas, playas, zonas montañosas o en los valles de los ríos.

- Debido a que los turistas no hablan necesariamente el idioma del país que están visitando la comunicación con ellos no es lo suficientemente rápida en el caso de la aparición de un suceso repentino. La creación de avisos entre turistas antes de visitar el país es una tarea necesaria

- Si los turistas llegan ser víctimas de un desastre natural el impacto negativo en la imagen del destino turístico puede llegar a ser serio y provocar grandes pérdidas.

\section{Naturaleza y fuentes de crisis y desastres}

Los términos crisis y desastre no son sinónimos y para algunos autores existen elementos esenciales que diferencian la gestión de crisis y gestión de desastre. Faulkner (2001: 136) propone el término crisis como "una situación en la que la causa raíz del suceso es provocada por la propia organización debido a una ineficiente gestión u organización, o a una deficiente adaptación al cambio". Por otro lado, entiende por desastre como una "situación en la cual una empresa, conjunto de empresas o un destino turístico se enfrenta a cambios catastróficos, repentinos e impredecibles, sobre los cuales se tiene escaso control". El término crisis parece excluir para Faulkner aquellas situaciones en las que la buena marcha de la organización se ve amenazada por sucesos sobre los cuales se tiene escaso o nulo control (Figura 1). Sin embargo, considera que las crisis pueden surgir o agravarse por una deficiente gestión de un desastre por lo que, a efectos prácticos, podemos considerar las crisis como el resultado de una catástrofe. Esta es el enfoque que empleamos en este estudio y que es compartido por Aktas y Gunlu (2005) cuando señalan tres elementos que determinan las situaciones que desembocan en una crisis:

- Un elemento provocador. Las crisis son el resultado de un suceso inesperado u sucesión de eventos que se desarrollan en un corto periodo de tiempo y con el potencial de provocar un cambio sustancial, desafiando la estructura existente o supervivencia del destino turístico.

Amenaza y daño. El suceso que provoca la crisis es tan significante en su impacto que podría causar pérdida de control entre los directamente afectados y podría suponer una amenaza en el corto plazo a las operaciones del destino.

Necesidad de acción. Con el fin de superar los efectos en el largo plazo, la crisis requiere acciones urgentes por medio de la cooperación de las principales autoridades y los stakeholders del sector. 


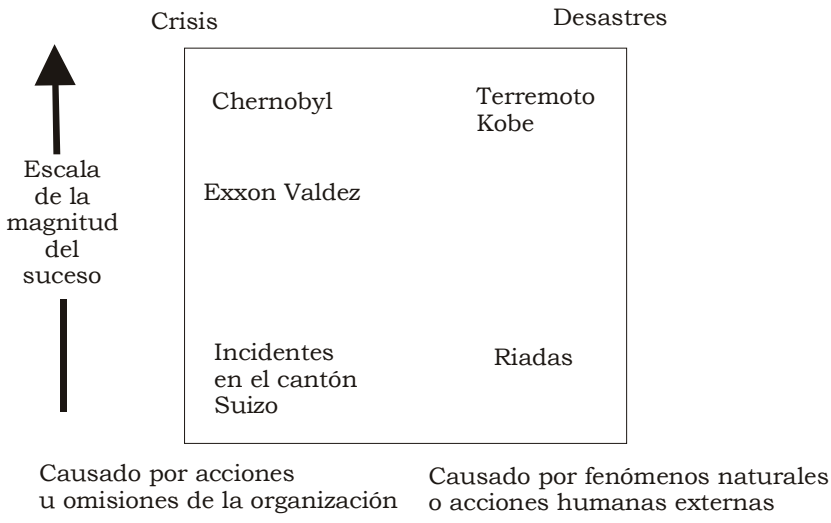

Figura 1. Concepto de crisis vs. desastre. Fuente: Faulkner (2001).

Por su misma naturaleza el turismo es un sector vulnerable a un rango de crisis más amplio que otros sectores. La aparición de crisis puede llegar a ser más frecuente, por ser una industria basada fundamentalmente en las expectativas y en la confianza, y por tratarse de un sector altamente susceptible a los factores externos y las presiones en el amplio entorno de operaciones. El conjunto de factores externos que le afectan es muy variado por ser un sector transversal con influencia en otros muchos sectores (Goeldner, Ritchie y Mcintosh, 2000). En el entorno de la industria turística mundial, el Foro Económico Mundial señala se pueden identificar tres principales fuentes de desastres (WEF, 2005): Medioambientales (por ejemplo, desastres naturales como terremotos, riadas, y provocados por el hombre como mareas negras e incendios), Geopolíticos (terrorismo) y Sociales (pandemias y epidemias).

En la misma línea, Luecke (2005: 4) presenta un breve esquema sobre cuáles son las fuentes de las crisis:

- Accidentes y hechos naturales

- Desastres sanitarios y medioambientales

- Manipulación de productos

- Accidentes catastróficos

- Daños medioambientales

- Fallos tecnológicos

- Fuerzas económicas y del mercado

- Malas prácticas de los empleados

Glaesser (2003) presenta un diagrama de acontecimientos negativos (Figura 2) en el que realiza una clasificación de eventos que afectan al turismo según el nivel de sorpresa o imprevisibilidad y el grado de control que tiene la organización o destino sobre ese suceso. Los acontecimientos con un sesgo más negativo serían aquellos con menor grado de control y mayor imprevisibilidad, por ejemplo, huracanes, terremotos, incendios o terrorismo. Esencialmente, cuando hablamos de una crisis o un desastre estamos refiriéndonos a un estado provocado por un suceso más bien imprevisto y sobre el cual tenemos poca capacidad de control, por lo que, en propiedad, serían los sucesos negativos situados en el cuarto cuadrante los que realmente serían fuentes o elementos provocadores de una crisis o un desastre.

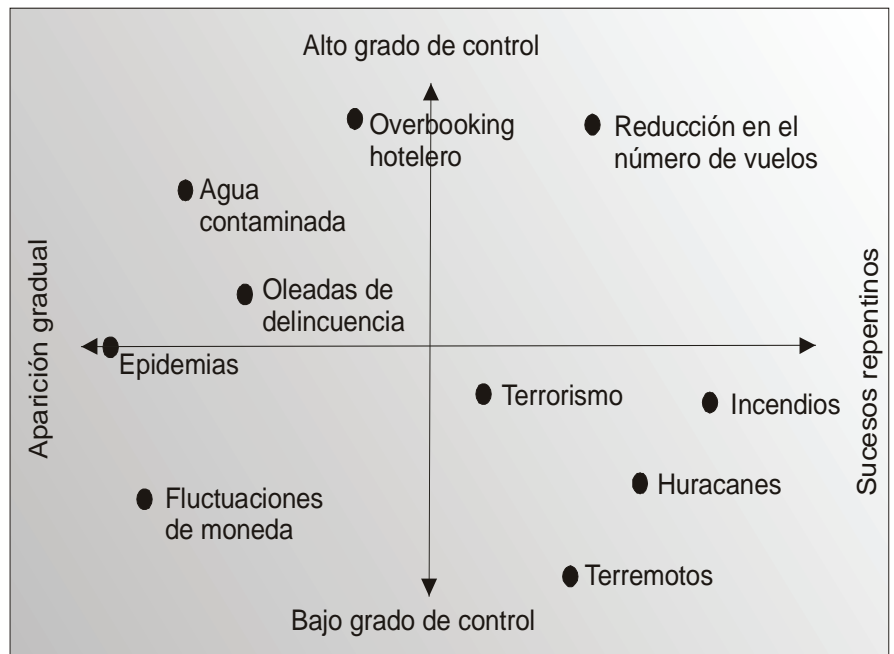

Figura 2. Diagrama de acontecimientos negativos. Fuente: Glaesser (2003: 22)

Henderson (2007) amplía esta visión y realiza una completa clasificación de las fuentes de las crisis diferenciado las amenazas externas de aquellas que se generan en la propia organización-destino situando cada una de las amenazas en el ámbito apropiado (social, económico, medioambiental, político, tecnológico y comercial) (Tabla 1). Existen amenazas que pueden encuadrase en varios dominios, por ejemplo la amenaza de incendios aparece tanto en el dominio de tecnológico (incendio en un hotel provocado por un cortocircuito) como en el dominio medioambiental (incendio forestal).

\section{Gestión de crisis y desastres, modelos proactivos}

La sensibilidad del turismo a un amplio abanico de factores externos no hace a la industria turística más débil que otras pues se ha comprobado con regularidad e históricamente que el sector turístico es una industria probada y resistente a las crisis, y su recuperación es rápida, llamativamente más rápida que otros sectores ante las grandes crisis (Pike, 2004). Podríamos decir que el turismo es un sector más propenso que otros a experimentar crisis, pero por sus características se recupera mejor y más rápido llegando a ser un elemento tractor de la recuperación. Para De Sausmarez (2007) el turismo es un sector ideal en el que apoyarse para em- 


\begin{tabular}{|c|c|c|}
\hline Dominio & Externo & Interno \\
\hline Económico & $\begin{array}{l}\text { Recesión } \\
\text { Fluctuaciones en la moneda } \\
\text { Impuestos }\end{array}$ & $\begin{array}{l}\text { Crecimiento de los costes } \\
\text { Caída de ingresos } \\
\text { Falta de beneficios }\end{array}$ \\
\hline Político & $\begin{array}{l}\text { Políticas del gobierno } \\
\text { Rela ciones interna cionales } \\
\text { Inestabilidad } \\
\text { Terrorismo }\end{array}$ & \\
\hline Socio-cultural & $\begin{array}{l}\text { Disturbios } \\
\text { Delincuencia } \\
\text { Xenofobia }\end{array}$ & Conflictos culturales \\
\hline Medioambiental & $\begin{array}{l}\text { Fenómenos natura les } \\
\text { Contaminación } \\
\text { Alarmas contra la salud }\end{array}$ & $\begin{array}{l}\text { Hiper-desa rrollo } \\
\text { Degra da ción } \\
\text { medioambiental }\end{array}$ \\
\hline Tecnológico & $\begin{array}{l}\text { Fallos en sistemas tecnológicos } \\
\text { Fallos de diseño } \\
\text { Incendios }\end{array}$ & Accidentes de transporte \\
\hline Comercial & Intervención del Gobierno & $\begin{array}{l}\text { Errores humanos } \\
\text { Decisiones directivas } \\
\text { Conflictos laborales } \\
\text { Competitividad } \\
\end{array}$ \\
\hline
\end{tabular}

Tabla 1. Amenazas externas e internas en la crisis. Fuente: Elaboración propia basado en Henderson (2007)

pezar los esfuerzos de recuperación de un territorio tras una crisis. Su estrecha conexión con otros sectores de la economía hace que sea más eficaz para el gobierno concentrar sus inversiones al principio en este sector antes que difuminarlas por varios sectores, consiguiendo así retornos de inversión más rápidos. Como recuerda el Foro Económico Mundial "una eficaz gestión de crisis puede hacer del sector turístico un catalizador de la recuperación general económica y social" (WEF, 2005: 5).

Las crisis y los desastres tienen connotaciones de transformación ya que los sucesos que las provocan poseen un gran potencial de cambio, con consecuencias positivas o negativas. Faulkner (2001) incide en el potencial positivo de las crisis, ya que pueden actuar como punto de inflexión -turning point-, presentando una oportunidad de mejora a las organizaciones y destinos. En este sentido, el tsunami que afectó al sudeste asiático en diciembre de 2006 ofreció una oportunidad para corregir errores del pasado en la sostenibilidad del turismo de los destinos turístico afectados, y permitió, en la fase de recuperación y restauración, mejorar estos aspectos y la distribución del producto turístico en el mercado internacional (WTO, 2005).

La incidencia de desastres y crisis se irá incrementado a lo largo del tiempo y, por lo tanto, es cada vez más perentoria la necesidad de poner en práctica medidas eficaces de gestión de crisis que contribuyan a una rápida y eficiente recuperación del destino después de la crisis. Sin embargo, al tratarse de un sector fragmentado y diverso, es difícil establecer la forma de gestión de crisis que debe llevarse a cabo en el sector turístico (De Sausmarez, 2004). Wilks y Moore (2003) presentan un modelo de gestión de riesgos y crisis en un destino turístico compuesto por cuatro fases principales denominado modelo de las $4 \mathrm{R}^{1}$ : reducción, preparación, respuesta y recuperación (ver Tabla 2). En la fase de reducción se deben detectar las primeras señales de peligro, en la preparación se realiza la planificación de la crisis y se forman al personal en gestión de crisis, en la fase de respuesta se ejecutan las operaciones y planes de comunicación y por último se desarrolla la fase de recuperación, cuyo fin principal es la restauración y procurar el retorno a la normalidad del destino $u$ organización. Un sistema eficaz de gestión de crisis se actualiza continuamente y redefine las dos primeras fases como preparación para las dos segundas.

\begin{tabular}{cl}
\hline $\begin{array}{c}\text { Reducción } \\
\text { Reduction }\end{array}$ & $\begin{array}{l}\text { Vigilancia de la crisis } \\
\text { Políticas de observa ción y vigilancia } \\
\text { Protocolos y procedimiento de opera ciones }\end{array}$ \\
\hline \multirow{2}{*}{$\begin{array}{c}\text { Preparación } \\
\text { Readiness }\end{array}$} & $\begin{array}{l}\text { Plan de Gestión de Crisis } \\
\text { Planifica ción del Turismo } \\
\text { Medidas de Salud y Seguridad }\end{array}$ \\
\hline \multirow{2}{*}{ Respuesta } & $\begin{array}{l}\text { Procedimientos de respuesta de emergencia } \\
\text { Investiga ción }\end{array}$ \\
& $\begin{array}{l}\text { Asistencia a las familias } \\
\text { Comunica ción }\end{array}$ \\
\hline \multirow{2}{*}{$\begin{array}{c}\text { Recuperación } \\
\text { Recovery }\end{array}$} & $\begin{array}{l}\text { Plan de empresa continuo } \\
\text { Recursos Humanos } \\
\text { Interrogatorio }\end{array}$ \\
\hline
\end{tabular}

Tabla 2. Gestión de crisis de la “4 R's”. Fuente: Wilks y Moore (2003).

En la fase de reducción Wilks y Moore (2003) señalan tres puntos clave a considerar: 1) Vigilancia de la crisis: identificar riesgos, peligros, amenazas y posibles impactos. Recolección de datos e información; 2) Políticas de observación y continuidad: políticas de cooperación y políticas de seguridad y 3) Protocolos y procedimiento de operaciones: anticipación de problemas, revisión de procedimientos y refuerzo del personal de vigilancia.

A la fase de reducción sigue la preparación. Uno de los pasos primeros pasos a dar por las organizaciones responsables de los destinos turísticos es evaluar los 
riesgos a los que un destino concreto está expuesto y desarrollar planes para gestionar las situaciones de desastre que puedan producirse en el futuro. Los responsables turísticos tienen que auditar los planes regularmente, dirigir ejercicios de respuesta a la crisis y procurar la adquisición de habilidades para enfrentarse a las crisis. Toda la cadena de autoridad debe formar parte del programa de preparación para la gestión de la crisis y debe llegar a convertirse en parte de la cultura de la empresa. Para muchos autores tan importante es la planificación como la ejecución, "los directivos de las organizaciones preparadas ante la crisis han llegado a ver y usar la gestión de crisis como una nueva herramienta para obtener ventajas competitivas" (Mitroff, Pearson y Harrison, 2002: 120). Para Johnson, Tolomiczenko, y Gellatly (2008) la fase de planificación y preparación es la más asequible y eficaz, pero al mismo tiempo la menos utilizada. Es en esta fase cuando se pueden tomar acciones en orden a prevenir o mitigar los efectos potenciales de la crisis: desarrollar el plan de crisis, trabajar con los diversos stakeholders del sector, constituir un equipo de gestión de crisis, gestionar la percepción de riesgos mediante una adecuada política de información para reducir la incertidumbre, revisar los planes estratégicos frecuentemente y evaluar la necesidad de cambio, establecer un proceso de culturización y entrenamiento para gestionar la crisis formal e informalmente a través de todos los niveles de la organización (Aguirre y Ahearn, 2007). Una mayor preparación facilita y asegura estos aspectos. Glaesser (2003) incide en la importancia de la fase de preparación cuando distingue las tres fases del proceso de crisis (Tabla 3). La prevención de crisis es una fase continua que comprende dos áreas independientes aunque desde una perspectiva temporal se podrían desarrollar simultáneamente: precauciones acerca de la crisis y evitar la crisis. Las precauciones frente a la crisis describen actividades de planificación y medidas para tratar con más eficacia la crisis cuando llegue y conseguir que el daño producido sea el mínimo posible. Se trata de un área de naturaleza estratégica e implica políticas de crisis y preparación operativa para planes de crisis. El segundo área, evitar la crisis, se centra en los cambios o ajustes que realiza la organización o destino para adaptarse mejor a la nueva situación e incrementar la velocidad de reacción.

\begin{tabular}{|c|c|c|c|c|}
\hline \multicolumn{4}{|c|}{ Prevención de la crisis } & Abordarla crisis \\
\hline Precaucion & s a cerca la crisis & Evitarla c & & \multirow{2}{*}{ Empleo de instrumentos } \\
\hline Planifica ción & Implementa ción & Primeros avisos & Ajustes & \\
\hline
\end{tabular}

Tabla 3. Fases de la gestión de crisis. Fuente: Glaesser (2003).
El turismo es un sector que vende sensaciones positivas y parece incongruente acometer actividades de planificación de crisis ya que se asocia este sector con los aspectos positivos de tranquilidad y entretenimiento que suele acompañar a las vacaciones, precisamente esos aspectos son los que inciden con mayor fuerza en la percepción de riesgos del turista y hacen que se convierta en un sector más vulnerable cuando se presenta una pequeña amenaza (González Herrero, 1998). En el ámbito de la gestión de crisis en organizaciones industriales Pauchant y Mitroff (1992) denominan gestión de crisis proactiva a los planes de prevención o de anticipación de la crisis frente a las acciones tomadas durante o después de la crisis, que denominan gestión de crisis reactivas. A pesar del crecimiento del número de desastres y de crisis que tienen un impacto negativo sobre el turismo no ha habido un correspondiente desarrollo de la formulación de planes proactivos contra estas crisis, hasta el punto que Hystad y Keller (2008) consideran mínimas las formulaciones proactivas. Otros autores inciden en este punto, así Pforr (2006) argumenta la particular importancia de poner en práctica estrategias proactivas. En su opinión, los gobiernos y asociaciones del sector turístico desempeñan un papel de liderazgo en los asuntos importantes de la gestión de crisis, como son la comunicación, la información y la confianza en el destino, sin embargo, este papel se ha orientado a una línea de gestión de crisis reactiva, como desarrollo de patrones de respuesta, o guías prácticas de cómo responder a una crisis.

Un modelo o sistema proactivo de gestión de crisis presenta una estrategia general que habrá de ser contrastada e implementada en las operaciones concretas de la gestión de la crisis. Este sistema es una aproximación de 'arriba abajo', centrándose en las responsabilidades e interacciones de los principales agentes y negocios del sector y como ven los negocios turísticos su contribución durante las distintas fases del desastre. Es principalmente en los últimos años, ya en el siglo actual, cuando diversos autores han propuesto modelos como el que apuntamos: Faulkner (2001), Ritchie (2004), De Sausmarez (2004), Evans y Elphick (2005) y Paraskevas y Arendell (2007). Estos modelos o sistemas de gestión de crisis pretenden aportar un marco sistemático de actuación frente a las crisis desde una perspectiva estratégica, abarcando de modo proactivo todos los agentes y elementos que son afectados por la crisis. Estos modelos no pueden servir sólo como marcos teóricos sino que se deben perfeccionar mediante su contraste en situa- 
ciones y casos reales de crisis y desastres. Este desarrollo práctico provocará una mejora y perfeccionamiento de los modelos y procurará una mejor preparación para que los stakeholders dispongan de mayor orientación en las operaciones y en la toma de decisiones frente a la crisis.

Dentro de la investigación realizada en la gestión de crisis turística destaca la utilización de la herramienta diseñada por Faulkner (2001) y más adelante desarrollada por Faulkner y Vikulov (2001). En la Figura 3 se presenta el modelo de gestión de desastres en el turismo de Faulkner y que es empleada en diversos estudios para analizar la respuesta a crisis de orígenes muy variados (Miller y Ritchie, 2003; Henderson y Ng, 2004 y Peters y Pikkemaat, 2005). Esta necesidad de enriquecimiento y desarrollo de los modelos conceptuales y teóricos por medio de su contraste con acontecimientos de crisis y desastres es apuntada como una de las tareas prioritarias y de mayor interés con el fin de examinar cuáles son los elementos que favorecen una gestión de crisis más eficiente y eficaz en la industria turística" (Ritchie, 2004). Para De Sausmarez (2004) una gestión de crisis proactiva no es siempre tan sencilla por una serie de razones: la identificación y aproximación a la crisis mediante el seguimiento de indicadores adecuados y con el tiempo suficiente para poder tomar medidas llega a ser tan compleja como irreal, por otra parte la posibilidad de la obtención de fondos puede ser un desafío de considerable importancia en el caso de países en desarrollo, y por último, puede que no exista una estructura institucional adecuada para desarrollar un modelo previo de gestión de crisis; un ineficiente sistema y una pesada burocracia puede ralentizar y dificultar la introducción de medidas proactivas. A pesar de todo De Sausmarez asegura que la gestión de crisis proactiva está poco es- tudiada en comparación con la investigación sobre recuperación o respuestas a la crisis.

\section{Entrevistas a responsables del turismo en Galicia}

En diversos estudios de gestión de crisis provocadas por acontecimientos negativos la metodología empleada en la investigación ha sido cualitativa (ver Tabla 4). Esto es debido a que existe poca investigación sobre el tema y todavía es necesario realizar una aproximación con el objetivo de afrontar el problema con mayor conocimiento (Campiranon y Arcodia, 2007 y Korstanje, 2010). También Evans y Elphick (2005) defienden la utilización de métodos de investigación cualitativa basado en el hecho de que, bajo su punto de vista, no hay dos crisis iguales.

Con el fin de abordar una primera aproximación a los aspectos de la crisis, de abril a mayo de 2008 se realizaron entrevistas en profundidad a doce responsables de promoción y comunicación de Organismos Regionales y Locales de Turismo de Galicia sobre la preparación, gestión y acciones de promoción desarrolladas antes, durante y después de una crisis. Se emplearon en la investigación cuestionarios semiestructurados con preguntas abiertas que permitieron a los entrevistados ampliar o matizar algunas respuestas, teniendo en cuenta que se estaba tratando un tema sensible, en el que el entrevistado podía tener la percepción de que se quería depurar responsabilidades o realizar una revisión crítica de su actuación durante el periodo de la crisis.

Los desastres sufridos en Galicia en los últimos años parecen motivo suficiente para abordar las entrevistas en este destino turístico, los impactos más importantes fueron el hundimiento del petrolero Prestige a unos 250 kilómetros de la costa de Galicia el 19 de noviembre de

\begin{tabular}{|l|l|l|}
\hline \multicolumn{1}{|c|}{ Estudio } & \multicolumn{1}{|c|}{ Suceso } \\
\hline Faulknery Vikulov (2001) & $\begin{array}{l}\text { Lluvias torrencia les y riadas en } \\
\text { Katherine (Austra lia) }\end{array}$ & $\begin{array}{l}\text { Entrevistas en profundidad. Utilizan una parrilla para la } \\
\text { eva lua ción de las respuestas a un suceso cata strófico (DIRE, } \\
\text { Disaster Incident Response Evaluation) y posteriormente un } \\
\text { workshop pa ra examen, va lora ción y análisis }\end{array}$ \\
\hline Evans y Elphick (2005) & 11-S y fiebre aftosa en Gran Bretaña & $\begin{array}{l}\text { Métodos de investiga ción cua lita tivos. Cuestiona rio } \\
\text { semiestructura do, enfoque deductivo. }\end{array}$ \\
\hline Cioccio y Michael (2007) & Incendios en Victoria (Austra lia) & Entrevistas en profundidad; enfoque narra tivo \\
\hline Burby y Wagner (1996) & Hura cán en Nueva Orleans (USA) & Entrevistas persona les \\
\hline Lo, Cheung y Law (2006) & Virus del SARS en Hong Kong & $\begin{array}{l}\text { Entrevistas en profundidad. Cuestiona rio semiestructurado, } \\
\text { cotejado con fuentes secunda rias }\end{array}$ \\
\hline Miller y Ritchie (2003) & $\begin{array}{l}\text { Fiebre aftosa en Cheltnham (Gran } \\
\text { Bretaña) }\end{array}$ & $\begin{array}{l}\text { Aplican el modelo de Faulkner (2001). Entrevistas con } \\
\text { cuestionario semi-estructurado }\end{array}$ \\
\hline
\end{tabular}

Tabla 4. Estudios cualitativos de casos de crisis en el turismo. Fuente: Elaboración propia 


\begin{tabular}{|c|c|c|}
\hline $\begin{array}{l}\text { Secuencia en el proceso de } \\
\text { desastre }\end{array}$ & $\begin{array}{l}\text { Elementos de reacción en la } \\
\text { gestión del desastre }\end{array}$ & $\begin{array}{l}\text { Principales elementos en la } \\
\text { gestión estratégica del desastre }\end{array}$ \\
\hline $\begin{array}{l}\text { 1.Pre-suceso } \\
\text { Se toman acciones para prevenir o } \\
\text { mitigar los efectos de desastres } \\
\text { potenciales }\end{array}$ & $\begin{array}{l}\text { Precursores } \\
\text { - Nombrar un equipo de gestión de } \\
\text { desastres y su jefe } \\
\text { - Identificar agencias y } \\
\text { organizaciones relevantes del } \\
\text { sector publico y privado } \\
\text { - Establecer un marco de } \\
\text { coordinación, consultas y sistemas } \\
\text { de comunicación } \\
\text { - Desarrollar, documentar y } \\
\text { comunicar una estrategia de } \\
\text { gestión contra desastres } \\
\text { - Educar a los empleados, } \\
\text { stakeholders, clientes y ciudadanía } \\
\text {-Acuerdo y responsabilidad para la } \\
\text { activación de los protocolos de } \\
\text { emergencia }\end{array}$ & $\begin{array}{l}\text { Evaluación de riesgos } \\
\text { - Valoración de desastres } \\
\text { potenciales y su probabilidad de } \\
\text { que ocurran } \\
\text { - Desarrollo de escenarios en su } \\
\text { génesis y el impacto de potenciales } \\
\text { desastres } \\
\text { - Desarrollo de planes de } \\
\text { emergencia frente a catástrofes }\end{array}$ \\
\hline $\begin{array}{l}\text { 2. Prodromo } \\
\text { El desastre en inminente }\end{array}$ & $\begin{array}{l}\text { Movilización } \\
\text { Sistemas de aviso (incluyendo los } \\
\text { medios de comunicación) } \\
\text { Establecer un centro de dirección } \\
\text { Servicios/equipamientos de } \\
\text { seguridad }\end{array}$ & \multirow{5}{*}{$\begin{array}{l}\text { Planes de emergencia por } \\
\text { catástrofe } \\
\text { - Identificar impactos probables y } \\
\text { grupos de riesgo } \\
\text { - Evaluar las capacidades de } \\
\text { residentes y visitantes para } \\
\text { afrontar los impactos } \\
\text { - Articular los objetivos de los } \\
\text { planes de emergencia individuales } \\
\text { (para desastres específicos) } \\
\text { - Identificar las acciones necesarias } \\
\text { para evitar o minimizar los efectos } \\
\text { de cada fase } \\
\text { - Idear un perfil de estrategias } \\
\text { prioritarias para cada fase } \\
\text { o Prodromo } \\
\text { o Emergencia } \\
\text { o Intermedia } \\
\text { O Recuperación a largo plazo } \\
\text { - Revisión y examen a la luz de: } \\
\text { O Experiencia } \\
\text { O Cambios en la estructura de la } \\
\text { organización y en el personal } \\
\text { O Cambios en el entorno }\end{array}$} \\
\hline $\begin{array}{l}\text { 3. Emergencia } \\
\text { Se sienten los efectos del desastre } \\
\text { y se han de tomar medidas para } \\
\text { proteger bienes y personas }\end{array}$ & $\begin{array}{l}\text { Acción } \\
\text { - Procedimientos de evacuación o } \\
\text { rescate } \\
\text { - Provisión de alojamiento y } \\
\text { alimentos } \\
\text { - Servicios y cuidados médicos } \\
\text { - Sistemas de seguimiento y } \\
\text { comunicación }\end{array}$ & \\
\hline $\begin{array}{l}\text { 4. Intermedia } \\
\text { Punto en el que las necesidades a } \\
\text { corto plazo de las personas han } \\
\text { sido atendidas y el principal foco de } \\
\text { actividad es restaurar los servicios y } \\
\text { la población a la normalidad }\end{array}$ & $\begin{array}{l}\text { Recuperación } \\
\text { - Sistema de seguimiento y } \\
\text { auditoría de daños } \\
\text { - Limpieza y restauración } \\
\text { - Estrategia de comunicación con } \\
\text { los medios de comunicación }\end{array}$ & \\
\hline $\begin{array}{l}\text { 5. Largo plazo (recuperación) } \\
\text { Continuación de la anterior fase, } \\
\text { aspectos que no pudieron ser } \\
\text { atendidos tan rápidamente se } \\
\text { atienden ahora. Auditoría, auto- } \\
\text { análisis, curación }\end{array}$ & $\begin{array}{l}\text { Reconstrucción y re-evaluación } \\
\text { - Reparar los daños de la } \\
\text { infraestructura } \\
\text { - Rehabilitación del medio } \\
\text { ambiente en las áreas dañadas } \\
\text { - Asesoramiento a las víctimas } \\
\text { - Recuperación de la confianza de } \\
\text { empresarios y consumidores, } \\
\text { desarrollar planes de inversión } \\
\text { - Reunión para incorporar } \\
\text { experiencias y aprendizaje, y } \\
\text { revisar las estrategias desarrolladas }\end{array}$ & \\
\hline $\begin{array}{l}\text { 6. Resolución } \\
\text { Se recupera la rutina o se establece } \\
\text { un estado más avanzado }\end{array}$ & Revisión & \\
\hline
\end{tabular}

Figura 3: Modelo de Faulkner para la gestión de desastres en el turismo. Fuente: Faulkner (2001) 
$2002^{2}$ y los incendios forestales que asolaron casi cien mil hectáreas de bosque durante 7 días de agosto de 2006. El objetivo principal de estas entrevistas era conocer como había sido la preparación de los organismos más importantes en la comercialización del turismo en una situación de crisis, cuál fue la respuesta y las consecuencias en la promoción del turismo debida a la crisis. En este trabajo nos hemos centrado en los aspectos de planificación y preparación para la crisis de las distintas entidades consultadas, y tratar de identificar cuáles son los elementos más problemáticos. La toma de datos de las entrevistas se realizó por medio de un cuestionario realizado al efecto, tomando como base un estudio publicado de la Organización Mundial del Turismo (Luhrman, 2005). El cuestionario constaba de 71 preguntas, de las cuales 22 correspondían al apartado de actuación y medidas puestas en marcha antes de entrar en la crisis en el ámbito de la promoción (18 preguntas) y sobre la disponibilidad de estudios de mercado (4 preguntas).

Ninguno de los responsables de las organizaciones consultadas manifestó haber previsto una partida presupuestaria o haber realizado una reserva de fondos para emergencias o crisis. Un responsable de un Patronato de turismo provincial señaló que para que se dé una crisis tienen que confluir varios factores, una desgracia no tiene porque acabar necesariamente en crisis. Pese a las experiencias de desastres en los últimos años no existe una planificación orgánica ante posibles nuevos acontecimientos negativos, se tiene la confianza que es difícil que pase otra vez, y si pasa ya se atenderá cuando llegue. La preparación más frecuente es el mantenimiento de una red de contactos a los que se puede llegar rápidamente en caso de necesidad urgente: sistemas de envío masivo de información y mensajes, servicio de newsletter a contactos del sector turístico, envíos masivos de información a empresas del sector con información puntual sobre novedades y actualidad. La plataforma web es muy utilizada para la transmisión actual y rápida de la información, pero explícitamente sólo una entidad difunde aspectos de seguridad por medio de la web, las demás organizaciones no realizan comunicación en materia de seguridad.

Todos los entrevistados manifiestan actuar con completa honestidad en sus promociones de manera que no se ofrecen productos más allá de las posibilidades del destino, se procura de esta manera ganar la confianza de usuarios y empresas turísticas que en una situación de crisis es fundamental. Al mismo tiempo, la propia naturaleza del turismo puede llegar a forzar la imagen del destino ya que, según un entrevistado "se actúa con honestidad en la promoción, pero la promoción va antes que el producto por lo que para crear expectativas hay que ir por delante".

En cuanto a la utilización antes de la crisis de estudios de mercado podemos decir que existe una estructura de estos estudios empleados por las diferentes organizaciones y generados por dos o tres fuentes. La mayoría de estos estudios se refieren a datos de ocupación y pernoctaciones, algunos más elaborados llegan a analizar la satisfacción de los usuarios. Es muy escasa la utilización de estos estudios como herramienta de análisis y planificación para la crisis, como pudiera ser la simulación de escenarios y de actividades de recuperación. Se obtienen los datos aunque no se realiza un estudio exhaustivo de ellos de manera no se analizan para la toma de decisiones, confiando aparentemente más en la experiencia y la intuición.

En la respuestas de las entrevistas se percibe una preocupación real por la falta de coordinación entre los distintos organismos e instituciones. Existen diferentes instituciones que actúan en la gestión de la crisis con diferente intensidad y protagonismo: los patronatos locales de turismo, las entidades autónomas provinciales dependientes de las Diputaciones, los organismos del gobierno autonómico y las sociedades de promoción y comercialización del destino. Existen, además, en Galicia, los Centros de Desarrollo Comarcal, algunos de ellos muy activos, arraigados a una división del territorio histórica o natural. El sentir general de las fuentes consultadas es la inoperabilidad en el objetivo de coordinar las grandes líneas turísticas. Al no existir un acuerdo anterior a la crisis en la definición de unos objetivos comunes difícilmente se conseguirá esa colaboración a la hora de plasmarlos en acciones concretas durante la crisis.

Parece oportuno señalar los diferentes puntos de vista que mantienen los responsables de las distintas instituciones, principalmente entre los organismos de la administración autonómica que tienen asumidas las competencias de la ordenación y promoción del turismo en Galicia y las sociedades de promoción de comercialización de turismo constituidas en su mayoría por capital público. En opinión de los responsables del gobierno autonómico se realizaron una serie de acciones promocionales especialmente dirigidas a contrarrestar los efectos del desastre en el turismo. Sin embargo, la mayoría de los organismos de comercialización turística de las distintas zonas consultados consideran que no se proyectaron actuaciones específicas de relevancia y se limitaron a continuar con la promoción que habían planificado antes de la crisis. Nuestra opinión ante esta paradoja es que las acciones promocionales, que evidentemente se realizaron, se diseñaron y proyectaron unilateralmente por los organismos del gobierno autónomo 
con una evidente falta de cooperación y coordinación, y sin contar con el punto de vista profesional de las sociedades de comercialización, limitando la actuación de estas instituciones a la función de mero altavoz.

Todos los encuestados manifestaron su acuerdo de que la coordinación es condición necesaria para la gestión del desastre y la asignatura pendiente. Existen intereses de todo tipo, políticos, culturales, históricos, geográficos que impiden alcanzar un consenso entre los diversos organismos, pero existe una opinión coincidente en que la cooperación y colaboración entre los actores principales es el enfoque más adecuado para el desarrollo y planificación de la industria turística. Siguiendo a Williams y Ferguson (2005: 362) podemos afirmar que "en tiempo de crisis la asociación ideal debería ser la colaborativa. Esta colaboración crea una alianza estratégica con un objetivo o una visión que genera una cultura comprometida con la resolución de problemas”.

\section{Conclusiones}

Las investigaciones académicas sobre la crisis en el turismo se han enfocado principalmente hacia las acciones de comunicación y promoción, sin embargo se ve necesaria una visión proactiva y completa de la gestión de crisis en el turismo y con mayor incidencia, si cabe, en la preparación y la planificación. Una gestión estratégica eficaz de situaciones de crisis catastróficas en zonas turísticas puede generar incluso corrientes positivas en la demanda turística. La cooperación y coordinación entre las instituciones y agentes implicados aparece como la mejor herramienta para la planificación y posterior resolución de la crisis.

Es necesaria la planificación con unas líneas maestras de actuación comunes y consensuadas previamente que deberían respetarse por las partes implicadas, superando de esta manera el problema de las competencias. Un órgano representante y aglutinante de todos los agentes del sector sería el responsable de la definición de los planes y acciones concretas que dimanarían de ese plan de crisis para el turismo, adecuadamente alineado con el plan estratégico del sector. La preparación no sólo favorece a una mejor gestión en casos de crisis, sino que también favorece la integración y cohesión de los distintos agentes e instituciones del sector y una política de promoción más adecuada y más activa por parte de los organismos responsables de llevarla acabo. Pero no esta claro qué organismos son estos, cuáles han de tener la misión de integrar y cohesionar a los distintos stakeholders y llevar a cabo la planificación efectiva de la crisis antes de que ésta aparezca.

Tenemos la percepción de que las empresas y des- tinos que han realizado una planificación previa a la crisis tienen una posición de ventaja frente a otras para extraer o aprovechar las oportunidades que puede presentar una crisis. Si hemos de aprovechar oportunidades debemos conocer las cuáles son las medidas o prácticas más importantes y utilizadas en la promoción y recuperación de un destino tras una crisis con el fin de conocer su empleabilidad, esto requiere futuros estudios localizados en destinos concretos y orientados a los negocios del sector.

\section{Referencias bibliográficas}

Aguirre, J. A. y Ahearn, M.

2007 "Tourism, volcanic eruptions, and information: lessons for crisis management in National Parks. Costa Rica, 2006”. Pasos, Revista de Turismo y Patrimonio Cultural, 5(2): 175-191.

Aktas, G. y Gunlu, E. A.

2005 "Crisis management in tourist destinations". En W. F. Theobald (Ed), Global Tourism (pp 440-457). Amsterdam: Elsevier.

Beirman, D.

2003 Restoring Tourism destinations in crisis. A Strategic Marketing approach. Sydney: CABI Publishing.

Burby, R. J. y Wagner, F.

1996 "Protecting tourists from death and injury in coastal storms". Disasters, 20(1): 49-60.

Campiranon, K. y Arcodia, C.

2007 "Market segmentation in time crisis: A case study of the MICE sector en Thailand”. Journal of travel \& Tourism Marketing, 23 (2,3,4): 151-161.

Cioccio, L. y Michael, E. J.

2007 "Hazard or disaster: Tourism management for the inevitable in Northeast Victoria”. Tourism Management, 28: 1-11.

De Sausmarez, N.

2004 "Crisis Management for the Tourism Sector: Preliminary Considerations in Policy Development”. Tourism and Hospitality Planning \& Development, 1(2): 157-172.

De Sausmarez, N.

2007 "The potential for tourism in post-crisis recovery: lessons from Malaysia's experience of the Asian financial crisis”. Asia Pacific Business Review, April, 13 (2): 277-299.

Evans, N. y Elphick, S.

2005 "Models of Crisis Management: and Evaluation of their Value for Strategic Planning in the International Travel Industry”. International Journal of Tourism Research, 7: 135-150. 
Faulkner, B.

1999 Tourism disasters: towards a generic model. Cooperative Research Centre for Sustainable Tourism. CRC Tourism work-in-progress report series, $n^{\circ} 6$.

Faulkner, B.

2001 "Towards a framework for tourism disaster management”. Tourism Management, 22(2): 135-147.

Faulkner, B. y Vikulov, S.

2001 "Katherine, washed out one day, back on track the next: A post mortem of a tourism disaster". Tourism Management, 22(4): 331-344.

Glaesser, D.

2003 Crisis Management in the Tourism Industry. Oxford: Elsevier Butterworth-Heinemann.

Goeldner, C. R.; Ritchie, J.R. y Mcintosh, R. W.

2000 Tourism. Principles, Practices Philosophies. New York: John Wiley \& Sons, Inc.

González Herrero, A.

1998 "Comercialización de productos y planificación de crisis en el sector turístico: imagen corporativa y marketing centrado en el consumidor". Papers de Turisme, 24: 6-45.

Henderson, J.C.

2007 Tourism Crises: Causes, Consequences and Management. Amsterdam: Butterworth-Heinemann.

Henderson, J. C. y Ng, A.

2004 "Responding to Crisis: Severe Acute Respiratory Syndrome (SARS) and Hotels in Singapore”. International Journal of Tourism Research, 6: 411-419.

Hystad, P. W. y Keller, P. C.

2006 'Disaster management: Kelowna tourism industry's preparedness, impact and response a 2003 major forest fire". Journal of Hospitality and Tourism Management, 13 (1): 44-58.

Johnson T.; Lu, Z.; Tolomiczenko, G. y Gellatly, J.

2008 "SARS: lessons in strategic planning for hoteliers and destinations marketers". International Journal of Contemporary Hospitality Management, 20(3): 332-346.

Korstanje, M.

2010 "El 11 de septiembre y la teoría de la percepción de riesgo". Pasos, Revista de Turismo y Patrimonio Cultural, 8 (2): 389-402.

Lerbinger, $\mathrm{O}$.

1997 The crisis Manager: Facing a Risk and Responsibility. Mahwah, NJ: Lawrence Erlbaum Associates.

Lo, A; Cheung, C. y Law, R.

2006 "The survival of hotels during disaster: A case stu$d y$ of Hong Kong in 2003". Asia Pacific Journal of Tourism Research, 11(1): 65-80.

Luecke, R.

2005 Gestión de crisis. Convertirlas en oportunidades.
Barcelona: Harvard Business Essential-Deusto.

Luhrman, D.

2005 Crisis guidelines for the tourism industry. Acceso en abril de 2005 en la web de la Organización Mundial del Turismo: http://www.world-tourism.org/ market_research/recovery/Crisis and Disaster Management Guidelines.pdf

Mckercher, B. y Young, I.

1999 "The potential impact of the Millennium Bug on tourism". Tourism Management, 20(4): 533-547.

Miller, G.A. y Ritchie B. W.

2003 "A farming crisis or a tourism disaster? An analysis of the food and mouth disease in the UK". Current Issues in Tourism, 6(2): 150-171.

Mitroff, I.; Pearson, C. M. y Harrison, L. K.

2002 Como gestionar una crisis. Barcelona: Gestión 2000.

Paraskevas, A. y Arendell, B.

2007 "A strategic framework for terrorism prevention and mitigation in tourism destinations". Tourism Management, 28: 1560-1573.

Pauchant, T. C. y Mitroff, I. I.

1992 Transforming de Crisis-Prone Organization: preventing Individual, Organizational Environmental Tragedies. San Francisco: Jossey-Bass.

Peters, M. y Pikkemaat, B.

2005 "Crisis management in Alpine winter sports resorts- the 1999 avalanche disaster in Tyrol". En Laws, E. y Prideaux, B. (Eds.), Tourism Crises: Management Responses and Theoretical Insight (pp. 9-20). New York: The Haworth Hospitality Press.

Pforr, C.

2006 "Tourism in Post-Crisis is Tourism in Pre-Crisis: A Review of the Literature on Crisis Management in Tourism". Working Paper Series, 2006-1, School of Management, Curtin University of Technology.

Pike, S.

2004 Destination Marketing Organizations. Oxford: Elsevier.

Richardson, B.

1994 "Crisis management and the management strategy: time to 'loop the loop'". Disaster Prevention Management, 3(3): 59-80.

Ritchie, B. W.

2004 "Chaos, crises and disasters: a strategic approach to crisis management in the tourism industry". Tourism Management, 25(6): 669-683.

Ritchie, J. R. B. y Crouch, G. I.

2003 The competitive destination: A sustainable tourism perspective.Wallingford, UK: CABI Publishing.

WEF

2005 Disaster Response: The Tourism Dimension. Study 
on the Feasibility of a Global Tourism. Disaster Communication Network. Volume I: Main Report. World Economic Forum \& World Tourist Organization, http:// www.weforum.org/en/media/ publications/ GlobalRiskReports/index.htm.

Wilks, J. y Moore, S.

2003 Tourism Risk Management for the Asia Pacific Region: An Authoritative Guide for Managing Crises and Disasters. Commonwealth of Australia, APEC International Centre for Sustainable Tourism.

Williams, C. y Ferguson, M.

2005 "Recovering from crisis. Strategic alternatives for leisure and tourism providers based within a rural economy". International Journal of Public Sector Management, 18(4): 350-366.

WTO

2005 Tsunami: One Year On - A Summary of the Implementation of the Phuket Action Plan. Madrid: World Tourism Organization.

WTO y WMO

1998 Handbook on Natural Disaster Reduction in Tourist Areas. Madrid: World Tourism Organization y World Meteorological Organization.

Rocha Neto, João Mendes, Et Al.

2006 "Brasília e seu entorno: Considerações sobre os desafios de metrópole Emergente". Revista Múltipla. Brasília, Ano XI - vol. 14 - n ${ }^{\circ}$ 20, junho de 2006. p. 103-117. Disponível em: <http://www.upis.br/multipla.htm>. Acesso em: 13 Jul. 2007.

Romancini, Sônia Regina; Martins, Eledir da Cruz.

2005 "As representações da natureza no imaginário cultural da região de Cuiabá-MT". In: Almeida, Maria Geralda. (Org.). Tantos Cerrados: Múltiplas abordagens sobre a biodiversidade e singularidade sociocultural. Goiânia: Ed. Vieira, p. 97-114.

Santos, Milton.

2005 A urbanização brasileira. São Paulo: EDUSP.

Sassen, Saskia; Roost, Frank

2001 "A cidade: Local estratégico para a indústria global do entretenimento. In: Espaço \& Debates: Revista de Estudos Regionais e Urbanos". São Paulo, Núcleo de Estudos Regionais e Urbanos, Ano XVII, nº 41, 2001, p. 66-74.

Schvasberg, Benny.

2003 "Tendências e problemas da urbanização contemporânea no Brasil". In: Castriota, Leonardo Bacci. (Org.). Urbanização brasileiras: Redescobertas. Belo Horizonte: C/Arte, p. 43-65.

Silva, Boanerges Candido da, Et al.

2007 "O potencial da 'Cidade de Pedras' para incrementar o Ecoturismo em Pirenópolis-GO”. Revista P@ rtes. São Paulo. Disponível em: <http://www.partes. com.br/turismo>. Acesso em: 05 jul. 2008.

Silva, Boanerges Candido da, Et al.

2007 "Pirenópolis: Turismo x Crescimento Urbano". Revista P@rtes. São Paulo, Disponível em: <http://www. partes.com.br/turismo>. Acesso em: 05 jul. 2008.
Silva, Boanerges Candido da

2008 "Demanda turística e tecnologia em Pirenópolis, Estado de Goiás”. Dissertação de Mestrado, Curitiba, UTFPR, 2008. Disponível em: < http://www.ppgte.ct.utfpr.edu.br/>.

Silva, Heliton Leal.

2003 "Desenvolvimento econômico e impacto socioambientais no sudoeste de Goiás". Revista Múltipla. Brasília, Ano VIII - vol. 9 - n ${ }^{\circ} 15$, dez. 2003, p. 129148. Disponível em: <http://www.upis.br/multipla. htm>. Acesso em: 13 nov. 2006.

Siqueira, Deis.

2003 "Turismo e religiosidade em Brasília". In: Montoro, Tânia Siqueira (Org.). Cultura do turismo: desafios e práticas socioambientais. Brasília: Thesauros, p. 67-102.

Soares, Beatriz Ribeiro; Bessa Kelly Cristine F. de Oliveira.

1999 "As novas redes do cerrado e a realidade urbana brasileira”. Universidade Federal de Goiás: Boletim Goiano de Geografia. Vol. 19 2, Ed. UFG, Goiânia, p. 11-34.

Theodoro, Susi Huff; Et. al.

2002 "Cerrado: O celeiro saqueado". In: Duarte, Maria Laura Goulart; Theodoro, Susi Huff (Orgs.) Dilemas do Cerrado: Entre o ecologicamente (in) correto e o socialmente (in) justo. Rio de Janeiro: Garamond, p. $145-176$.

Tulik, Olga.

2000 "Residências secundárias no Estado de São Paulo - Identificação de centros emissores de demanda”. In: Lage, B. H. G.; Milone, Paulo Cezar. (Orgs.). Turismo: Teoria e prática. São Paulo: Atlas, p. 196-205. Villaça, Flávio.

1998 "Espaço intra-urbano no Brasil". São Paulo: Studio Nobel.

\section{NOTAS}

1. Por las iniciales en inglés de las cuatro fases: Reduction, Readiness, Response, Recovery.

2. En total el Prestige arrojó al océano Atlántico una carga de 77.000 Tm. de fuel oil provocando una densa marea negra que afectó a las costas de Galicia, norte de Portugal, toda la cornisa cantábrica y la costa atlántica francesa.

Recibido:

$08 / 06 / 10$

Reenviado:

$03 / 11 / 10$

Aceptado:

$05 / 11 / 10$

Sometido a evaluación por pares anónimos 